\title{
Bispecific antibody targets multiple Pseudomonas aeruginosa evasion mechanisms in the lung vasculature
}

\author{
Ajitha Thanabalasuriar, ${ }^{1}$ Bas G.J. Surewaard, ${ }^{1}$ Michelle E. Willson, ${ }^{1}$ Arpan S. Neupane, ${ }^{1}$ Charles K. Stover, ${ }^{2}$ Paul Warrener, ${ }^{2}$ \\ George Wilson, ${ }^{2}$ Ashley E. Keller, ${ }^{2}$ Bret R. Sellman, ${ }^{2}$ Antonio DiGiandomenico, ${ }^{2}$ and Paul Kubes ${ }^{1}$ \\ 'Department of Physiology and Pharmacology, Snyder Institute for Chronic Diseases, University of Calgary, Calgary, Alberta, Canada. ${ }^{2}$ Medlmmune LLC, Gaithersburg, Maryland, USA
}

\begin{abstract}
Pseudomonas aeruginosa is a major cause of severe infections that lead to bacteremia and high patient mortality. $P$. aeruginosa has evolved numerous evasion and subversion mechanisms that work in concert to overcome immune recognition and effector functions in hospitalized and immunosuppressed individuals. Here, we have used multilaser spinning-disk intravital microscopy to monitor the blood-borne stage in a murine bacteremic model of $P$. aeruginosa infection. $P$. aeruginosa adhered avidly to lung vasculature, where patrolling neutrophils and other immune cells were virtually blind to the pathogen's presence. This cloaking phenomenon was attributed to expression of Psl exopolysaccharide. Although an antiPsI mAb activated complement and enhanced neutrophil recognition of $P$. aeruginosa, neutrophil-mediated clearance of the pathogen was suboptimal owing to a second subversion mechanism, namely the type 3 secretion (T3S) injectisome. Indeed, T3S prevented phagosome acidification and resisted killing inside these compartments. Antibody-mediated inhibition of the T3S protein PcrV did not enhance bacterial phagocytosis but did enhance killing of the few bacteria ingested by neutrophils. A bispecific mAb targeting both Psl and PcrV enhanced neutrophil uptake of $P$. aeruginosa and also greatly increased inhibition of T3S function, allowing for phagosome acidification and bacterial killing. These data highlight the need to block multiple evasion and subversion mechanisms in tandem to kill $P$. aeruginosa.
\end{abstract}

\section{Introduction}

Pseudomonas aeruginosa is an important opportunistic bacterial pathogen that causes life-threatening infections in critically ill and immunocompromised individuals. It is one of the most common causes of Gram-negative pneumonia in ventilated patients, is the principle pathogen responsible for persistent/chronic infections in the cystic fibrosis lung, and is a frequent colonizer of burn wounds, catheters, and medical devices (1). However, P. aeruginosa causes most deaths when it enters the bloodstream and disseminates. Specifically in the case of hospital-acquired pneumonia, $P$. aeruginosa has previously been reported to disrupt the lung barrier and enter into the bloodstream causing bacteremia, resulting in poor patient prognosis $(2,3)$. Further complicating these infections is the fact that $P$. aeruginosa is often multi-drug-resistant, likely contributing to its advanced innate resistance mechanisms. $P$. aeruginosa resistance has been reported for nearly all commonly used antibiotics (4-6). How P. aeruginosa avoids the innate immune system to survive in the bloodstream and disseminate to various organs is not well understood. In vitro, $P$. aeruginosa has the capacity to evade complement deposition, prevent neutrophil recruitment, and even have cytolytic effects on immune cells upon phagocytosis (7). However, which of these evasion or subversion mechanisms might

Authorship note: A. DiGiandomenico and P. Kubes contributed equally as co-senior authors.

Conflict of interest: Partial research support for this work was received from Medimmune, AstraZeneca.

Submitted: July 25, 2016; Accepted: March 2, 2017.

Reference information: / Clin Invest. 2017;127(6):2249-2261.

https://doi.org/10.1172/JCl89652. work in vivo remains unknown. Visualization of the behavior of the pathogen and how it evades immunity when it gains access to the very dynamic systemic circulation has not yet been accomplished.

Candidates contributing to successful subversion and/or evasion of the immune system in vitro include the type 3 secretion (T3S) injectisome and the unique Psl exopolysaccharide, respectively (8). The $P$. aeruginosa T3S injectisome is a major determinant of virulence, and its expression is frequently associated with acute invasive infections that have been linked to increased mortality in infected patients (9). The needle-like injectisome apparatus permits translocation of effector proteins from the bacterium into the host cell. To date, only 4 T3S effectors have been functionally characterized for P. aeruginosa (ExoY, ExoS, ExoT, ExoU), which is far fewer compared with other well-characterized T3S injectisomes (e.g., Salmonella enterica SPI-1 has 13; Shigella species have 25; ref. 9), potentially making $P$. aeruginosa more amenable for targeted therapeutic intervention. However, T3S expression on the bacterial surface is highly regulated and may only occur at certain stages during infection, making it difficult to inhibit this crucial virulence factor of $P$. aeruginosa (10). There have been some in vitro studies showing that T3S injectisome is expressed upon host cell contact, in low-calcium environments, and in acidic $\mathrm{pH}$ environments $(10,11)$. The T3S injectisome has been shown to aid $P$. aeruginosa colonization of the host in a variety of ways, including systemic spread of the pathogen and the sequestration of P. aeruginosa in nonacidic vacuoles in corneal epithelial cells (12-14). Psl exopolysaccharide, on the other hand, is a serotype-independent and abundantly expressed extracellular sugar polymer implicated in $P$. aeruginosa biofilm formation (15). Psl has been implicated in preventing complement deposition, which would prevent macro- 
phage and neutrophil recognition of the bacteria $(15,16)$. Indeed, $P$. aeruginosa Psl prevented phagocytosis and oxidant production in neutrophils (16). However, to date, all of these studies have been performed in in vitro settings. Therefore we sought to see the subversion or evasion of immune cells by $P$. aeruginosa in an in vivo model. We aimed to further understand the spatiotemporal mechanism of $P$. aeruginosa pathogenesis and delineate how we can use antibody-based therapeutics to optimize $P$. aeruginosa eradication.

In this study, we aimed to understand the neutrophil-P. aeruginosa axis in vivo during a bacteremic model of infection using multilaser spinning-disk intravital microscopy to track the pathogen in the very dynamic bloodstream. We specifically looked at the blood-borne stage of infection because, regardless of the mode of entry of the pathogen, through the lung via pneumonia, through skin burns, or directly into the blood via a contaminated catheter, the majority of patients die from bacteremic sepsis (2). We also examined isogenic knockouts of Psl exopolysaccharide and PcrV component of the T3S injectisome. PcrV is necessary for T3S translocation; therefore mutant bacteria fail to have functional T3S and cannot deliver important toxins (10). P. aeruginosa was observed to be adequately eradicated from blood via intravascular macrophages in the liver (Kupffer cells). However, in stark contrast, the lung vasculature, which lacks intravascular macrophages, functioned as a safe haven and was subject to invasion by this pathogen via direct binding to the endothelium lining the blood vessels. Despite the presence of intravascular neutrophils patrolling the lung vasculature, these immune cells failed to detect and phagocytize $P$. aeruginosa binding to the endothelium, an event not seen with Staphylococcus aureus, Streptococcus pneumoniae, or Escherichi coli strains. While knockout of Psl exopolysaccharide expression greatly increased neutrophil-pathogen interactions through a complement-dependent mechanism, suboptimal intracellular bacterial killing activity was observed. Disruption of the T3S injectisome did not improve neutrophil capture of bacteria, but it significantly enhanced intracellular killing of $P$. aeruginosa in the few neutrophils that did take up the bacteria. Intriguingly, the bispecific mAb MEDI3902 (currently in phase IIb clinical testing; ref. 17), targeting PcrV and Psl, bound the surface of bacteria, enhancing neutrophil capture of the pathogen and thereby giving the antibody greater access inside the phagosome for subsequent inhibition of toxin injection, markedly improving eradication of $P$. aeruginosa. MEDI3902's protective function was enhanced more than with administration of individual parenteral anti-PcrV and anti-Psl mAbs in combination. These data suggest that a multispecific sequential approach to disarm important coordinated bacterial virulence mechanisms is necessary for efficient immune cell clearance of $P$. aeruginosa. We suggest that making use of $\mathrm{mAb}$ binding to abundant Psl exopolysaccharide is advantageous to deliver more antibody intracellularly, thereby more efficiently inhibiting intracellular events mediated by the T3S injectisome.

\section{Results}

The liver Kupffer cells catch and phagocytize P. aeruginosa optimal$l y$. Once $P$. aeruginosa has entered the bloodstream, 2 of the major organs that are involved in systemic bacterial infections are the liver and lung (18). We hypothesized that $P$. aeruginosa localization to the liver occurs because this organ has a resident population of intravascular macrophages known as Kupffer cells that reside in the sinusoids and are able to catch bacteria out of the mainstream of blood. Following i.v. infection of mice with $P$. aeruginosa that stably expresses GFP ( $P$. aeruginosa-GFP), we observed that these Kupffer cells initially catch or capture very fast-moving bacteria (under high shear conditions) out of the mainstream of blood, an unnecessary step in vitro under static conditions but absolutely critical in vivo in rapidly flowing blood (19). After capture, the Kupffer cells then rapidly phagocytize $P$. aeruginosa-GFP (Supplemental Video 1; supplemental material available online with this article; https://doi.org/10.1172/JCI89652DS1). P. aerugino$s a$ were selectively captured by Kupffer cells but did not bind to the sinusoidal endothelium or other cells (Supplemental Video 1). Within 3 hours after infection the GFP signal from P. aeruginosa was reduced by more than $50 \%$ of values seen at 15 minutes in the liver, and fewer Kupffer cells could be seen with bacteria in them (Figure 1A). The dissipation of the $P$. aeruginosa-GFP signal as noted by fewer Kupffer cells with bacteria at 3-4 hours (Figure 1A) suggests that the majority, but not all, of the bacteria are effectively killed in the liver. We previously observed that $S$. aureus-GFP actually replicated inside Kupffer cells and its fluorescent signal became much stronger as the colonies grew for 8-24 hours (20), whereas $P$. aeruginosa did not form larger colonies.

Both Psl and PcrV systems have been implicated in potential immune evasion by $P$. aeruginosa $(14,16)$. We next used isogenic mutant strains lacking Psl expression $(\triangle p s l A)$ and PcrV mutants lacking a functional T3S injectisome $(\Delta p c r V)$ to understand the role of these bacterial components during infection in vivo. Following i.v. infection of mice with WT-, $\triangle p s l A-$, or $\Delta p c r V$-GFP strains, we found that all 3 strains were captured out of the mainstream of blood and phagocytized equally well by Kupffer cells in the liver (Figure 1, B and C). However, while the WT and $\Delta p s l A$ strains were cleared equally well as noted by the dissipation of the GFP signal within Kupffer cells at 3-4 hours, the rate of Kupffer cell clearance for the $\Delta p c r V$ strain appeared to be much faster (Supplemental Figure 1A). At the same time point the number of Kupffer cells containing $P$. aeruginosa-GFP $\Delta p c r V$ was reduced by more than half compared with $\triangle p s l A$ or WT strains (Supplemental Figure 1A). To confirm these results, livers from mice infected with the 3 strains were harvested, and CFU of $P$. aeruginosa revealed the greatest killing in the $\triangle p c r V$ strain, indicated by low CFU (Supplemental Figure 1B). These results are consistent with previous reports indicating that T3S-deficient strains are avirulent in preclinical animal infection models and are rapidly cleared by the host innate immune system (21). Prophylactic immunization with anti-Psl and anti-PcrV mAbs did not improve the initial capture of WT $P$. aeruginosa by liver Kupffer cells (Figure 1D). Liver bacterial burden of $P$. aeruginosa at 6 hours reflected what was observed in animals infected with mutant bacteria, where the antibodies against PcrV but not Psl alone expedited eradication (Supplemental Figure 2).

Lung resident neutrophils do not detect $P$. aeruginosa. In contrast to the liver, rodent and human lungs lack intravascular macrophages but have an abundant population of intravascular neutrophils (22). In fact, these are one of the key phagocytic cells in the lung capillaries (23). We observed by flow cytometry that $5 \%-15 \%$ of CD45-positive cells in the lungs are neutrophils at steady state 
A

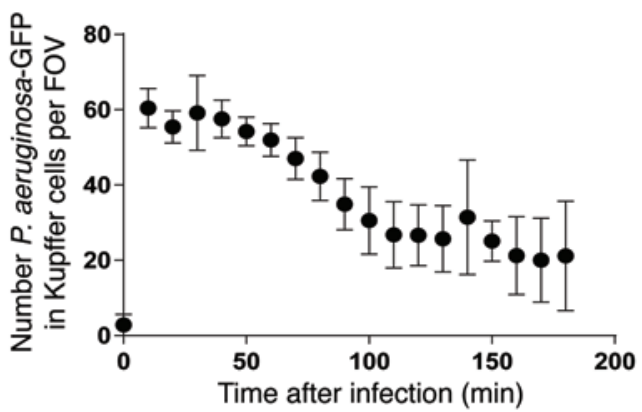

B
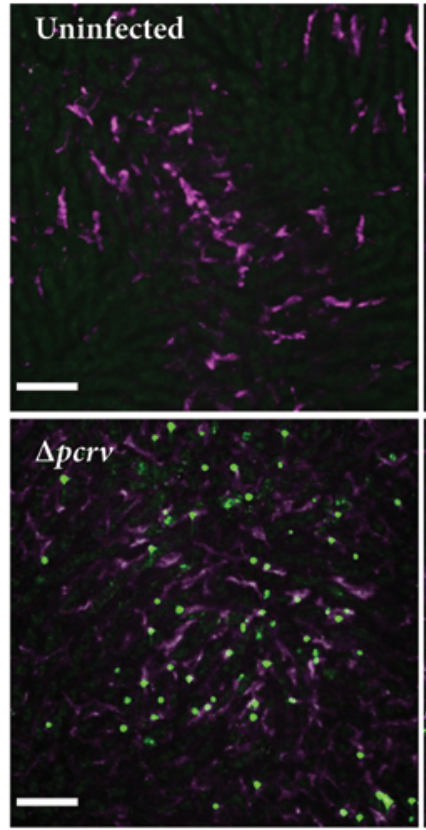

$P$. aeruginosa
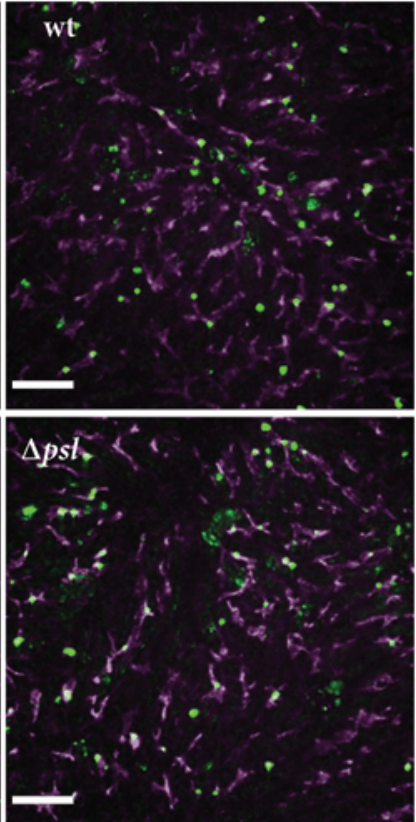

C

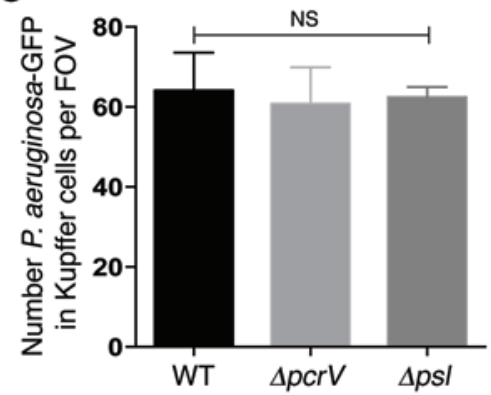

D

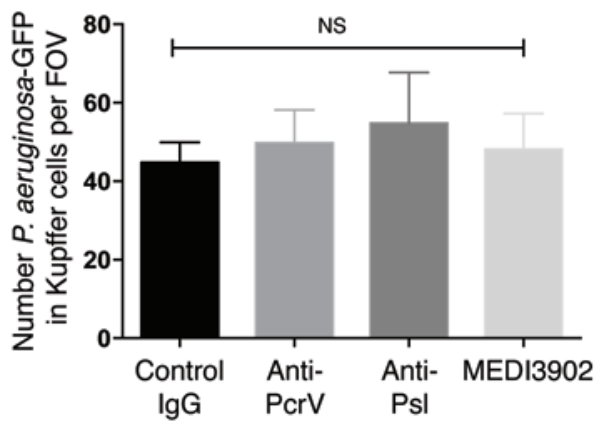

Figure 1. $\boldsymbol{P}$. aeruginosa is captured by liver Kupffer cells from the circulation. (A) Quantification of $P$. aeruginosa phagocytosis by Kupffer cells in the livers of mice. The number of bacteria arrested on or inside a Kupffer cell per field of view (FOV). $n=4$; 3 FOV per group were assessed. Error bars represent SEM. (B) Representative spinning-disk intravital microscopy (SD-IVM) image of liver 15 minutes after i.v. infection with $P$. aeruginosa (GFP) WT, $\triangle p s$ IA, or $\Delta p c r V$. Scale bars: $50 \mu \mathrm{m}$. (C) Quantification of SD-IVM images for number of Kupffer cells associated with $P$. aeruginosa-GFP was assessed at 15 minutes after i.v. infection with WT, $\triangle p s I A$, or $\Delta p c r V$ strains. $n=4 ; 3$ FOV per group were assessed. (D) WT mice treated 24 hours before i.v. infection with $15 \mathrm{mg} / \mathrm{kg}$ of control IgG, anti-Psl, anti-PcrV, or MEDI3902. Quantification of SD-IVM images for number of Kupffer cells associated with $P$. aeruginosa-CFP assessed at 15 minutes after i.v. infection with WT P. aeruginosa. Error bars represent SEM. One-way ANOVA was performed to determine statistical differences between groups. All experiments were repeated 3 times unless otherwise indicated.

(data not shown). Using intravital microscopy, we observed a resident population of neutrophils as they moved through the lung vasculature, tethering to the endothelium and then letting go or briefly adhering or crawling in the pulmonary capillaries (Supplemental Video 2). Upon i.v. infection of animals with P. aeruginosa, the bacteria firmly adhered to the lung capillaries often in close proximity to the intravascular neutrophils (Figure 2A). However, this influx of $P$. aeruginosa failed to be recognized by the neutrophils, and it was extremely rare to see neutrophils interact with and phagocytose the adherent bacteria (Figure 2A and Supplemental Video 3). This was surprising and unexpected, as intravascular infection of animals with other bacteria such as $S$. aureus, S. pneumoniae, or E. coli resulted in similar adhesion of the bacteria to the pulmonary capillaries and immediate engulfment of bacteria by the neutrophils (Figure 2, A and B, and Supplemental Video 4).
These data suggest that unlike other pathogens, $P$. aeruginosa may be able to escape neutrophil recognition in the lung vasculature.

Given the known presence of common pathogen-associated molecular patterns on the surface of $P$. aeruginosa, it was surprising to see a lack of neutrophil recognition after infection. As such, $P$. aeruginosa must employ a cloaking mechanism. The Psl exopolysaccharide has been previously postulated to restrict complement deposition onto bacterial membranes in vitro (16). Accordingly, we found that the $\triangle p s l A$, but not the $\Delta p c r V$, bacteria were readily recognized and phagocytized by lung intravascular neutrophils with increased efficiency compared with WT P. aeruginosa (Figure 2C). These data were confirmed by ex vivo quantification of $P$. aeruginosa-GFP-positive neutrophils by flow cytometry (Figure 2D). To determine whether complement was responsible for increased uptake of the psl mutant by neutrophils, animals deficient in com- 
A
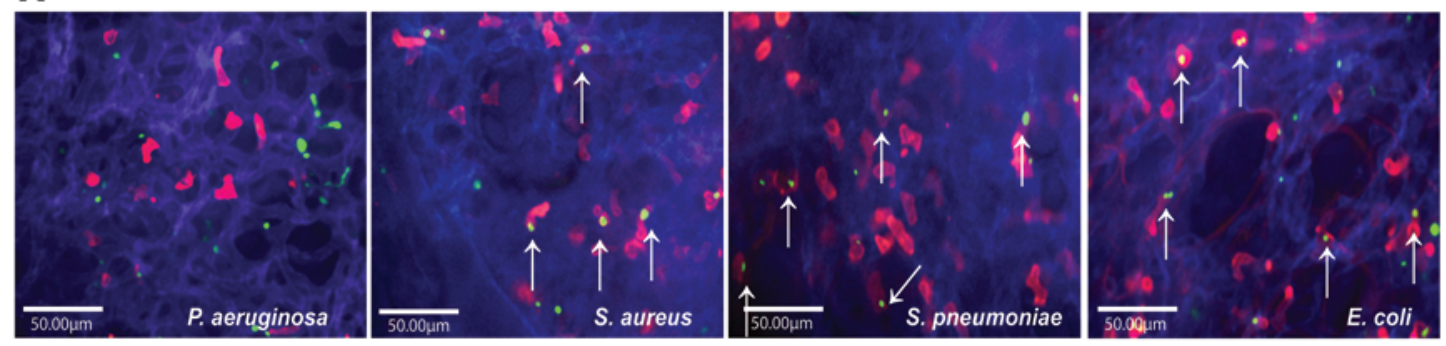

B

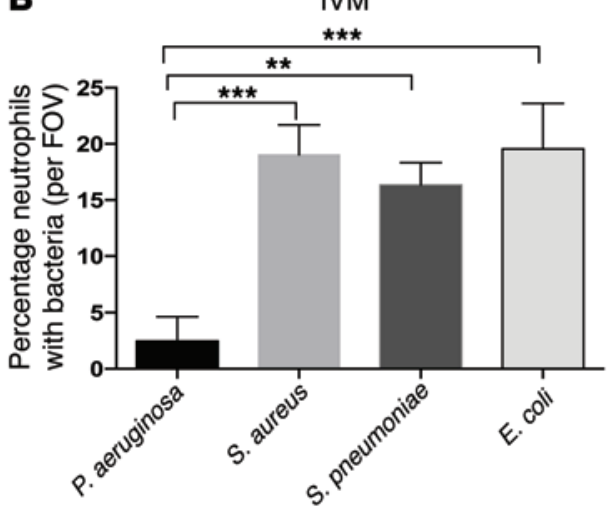

D

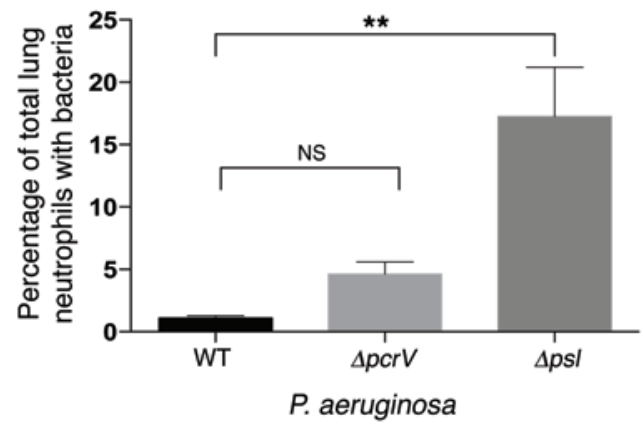

C

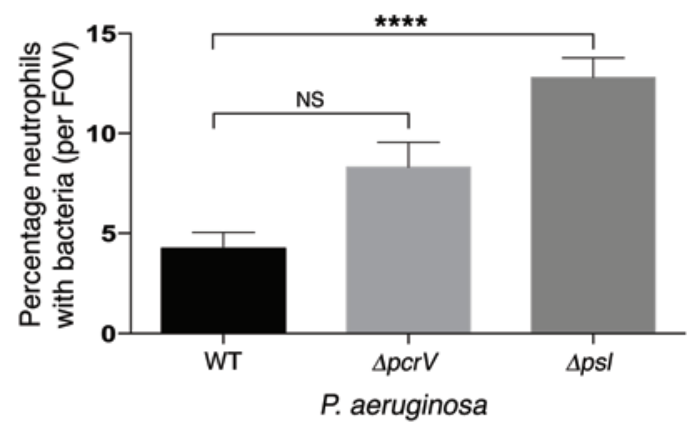

IVM

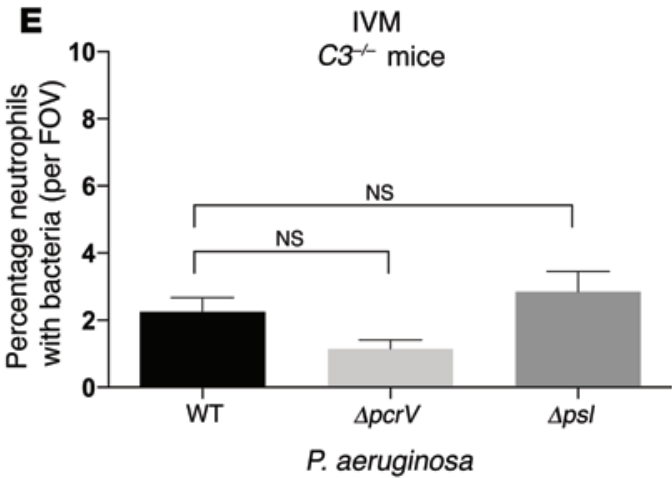

Figure 2. P. aeruginosa escapes lung vasculature neutrophil recognition via the Psl exopolysaccharide. (A) Still images of lung taken 20 minutes after i.v. infection by the specified pathogens using spinning-disk intravital imaging. Neutrophils, red; $P$. aeruginosa, S. aureus, S. pneumoniae, and $E$. coli, green; endothelial cells, blue. Scale bar: $50 \mu \mathrm{m}$. (B) Quantification of 10-minute intravital videos: percentage of neutrophils with bacteria per FOV was assessed in each mouse. $n=3$; 3 fields were assessed per group. Error bars represent SEM. ${ }^{* *} P=0.0006,{ }^{* *} P=0.0002$. (C) SD-IVM was used to assess the ability of pulmonary intravascular neutrophils to recognize the $P$. aeruginosa mutants $\Delta p s / A$ and $\Delta p c r V$ in the lung capillaries after blood-borne infections. Images represent still captures of live videos 20 minutes after infection. Graphs represent quantification of intravital videos: number of neutrophils with bacteria per FOV was assessed in each mouse. $n=4$; 3 fields were assessed per group; 10 -minute videos. Error bars represent SEM. ${ }^{* * * *} P<0.0001$. (D) Neutrophil phagocytosis of $P$. aeruginosa WT and mutants was assessed by flow cytometry. Percentage of Ly6G-positive cells containing $P$. aeruginosa-GFP. $n=3$. ${ }^{* *} P=0.0087$. (E) SD-IVM was used to assess the ability of pulmonary intravascular neutrophils to recognize $P$. aeruginosa mutants noted on the figure in the lung capillaries after blood-borne infections of complement component 3-knockout mice $\left(C 3^{-/}\right) . n=3 ; 3$ fields were assessed per group. Error bars represent SEM. Unless otherwise stated, 1-way ANOVA statistical analysis was performed on data points; multiple comparisons were performed between indicated columns. Graphs labeled "IVM" quantify intravital microscopy data, not flow cytometry data. All experiments were repeated 3 times unless otherwise indicated.

plement component $3\left(\mathrm{C3}^{--}\right)$were next examined. In these mice, neither WT, $\triangle p s l A$, nor $\triangle p c r V P$. aeruginosa strains were recognized by neutrophils (Figure 2E). These data suggest that Psl prevents complement activation or deposition on the surface of WT P. aeruginosa and this is critical for this bacterium to escape recognition by the intravascular lung resident neutrophil population.

Anti-Psl antibodies enable acute recognition of $P$. aeruginosa by neutrophils, while anti-PcrV anticytotoxic activity allows acidification of endosomal compartments. To determine whether we could over- come the evasion mechanisms of $P$. aeruginosa in the lung using therapeutic antibodies, mice were pretreated with mAbs targeting the Psl exopolysaccharide (anti-Psl) or the T3S injectisome PcrV component (anti-PcrV). In animals treated with the anti-Psl mAb, neutrophils recognized $P$. aeruginosa 3 - to 5 -fold above control IgG as indicated by neutrophils with internalized bacteria (Figure 3, A and B, and Supplemental Videos 5-7). These results are consistent with the increased neutrophil engagement and phagocytosis observed in experiments using Psl-deficient ( $\triangle p s l A)$ P. aeruginosa 
A

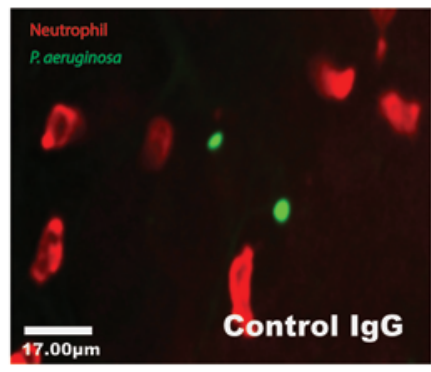

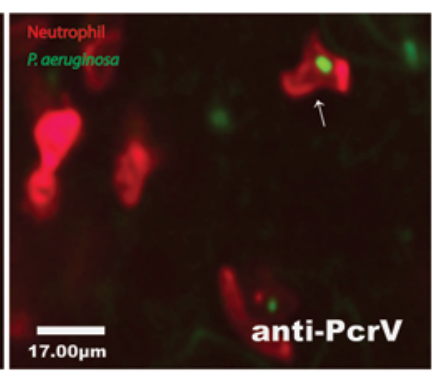

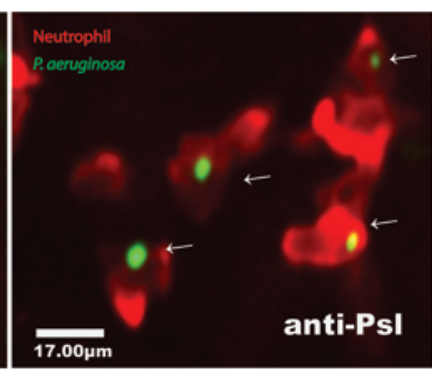

B

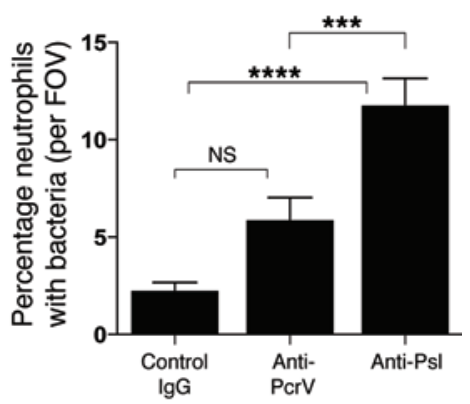

Antibody treatment
C

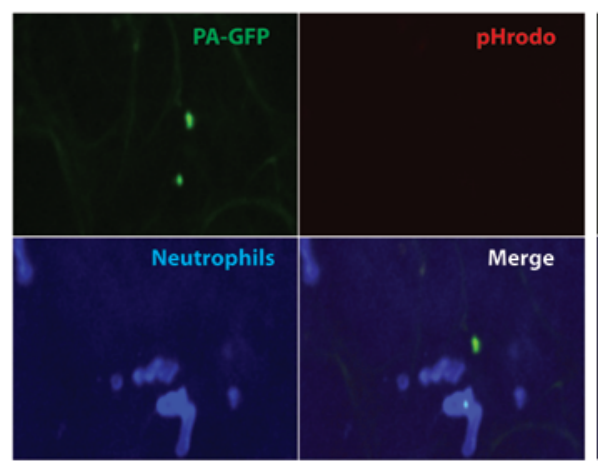

D

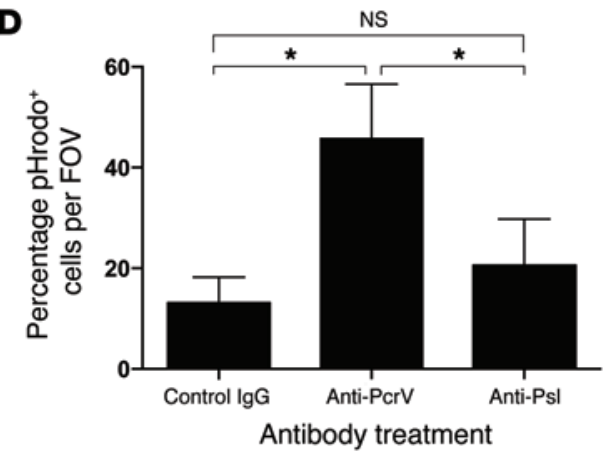

Anti-PcrV

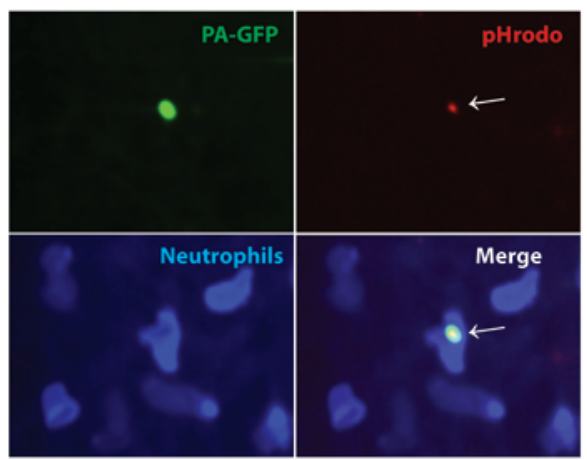

Anti-Psl

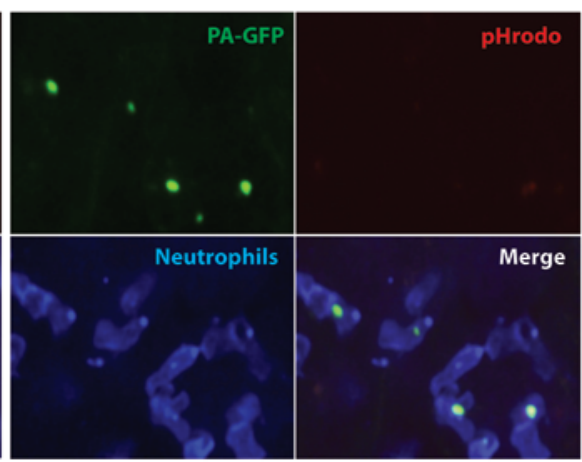

E

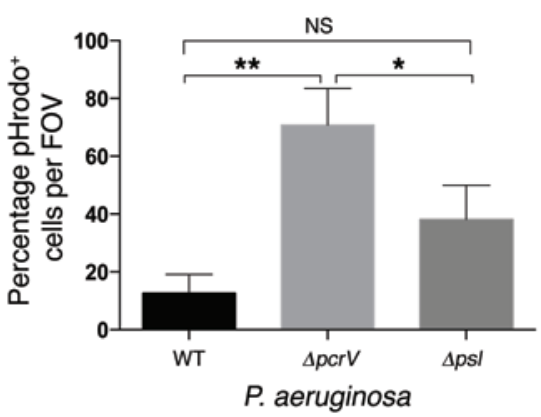

Figure 3. Anti-PsI antibodies enable acute recognition of $P$. aeruginosa, while Anti-PcrV anticytotoxic activity allows acidification of endosomal compartments. (A) SD-IVM was used to assess the ability of pulmonary intravascular neutrophils to recognize $P$. aeruginosa after blood-borne infections in the presence or absence of prophylactic antibody treatment. Neutrophils, red; $P$. aeruginosa, green. White arrows identify neutrophils containing bacteria. Representative images were captured 30 minutes after infection. (B) Quantification of intravital videos: number of neutrophils with bacteria per FOV was assessed in each mouse (3 FOV per mouse). $n=4$ per group; 10 -minute videos. Error bars represent SEM. ${ }^{* * *} P=0.0008,{ }^{* * *} P<0.0001$. (C) $P$. aeruginosa-GFP stained with pHrodo, a dye that increases in red signal when it is in an acidic environment. Neutrophils, blue; $P$. aeruginosa-GFP stained with pHrodo, green and red. White arrows identify pHrodo-positive cells. (D) Quantification of pHrodo-positive neutrophils per FOV. Three FOV were assessed in 4 mice. Error bars represent SEM. ${ }^{*} P=0.03$. (E) Quantification of pHrodo-positive neutrophils in mice infected with respective $P$. aeruginosa mutants. Three FOV were assessed in 4 mice. Error bars represent SEM. ${ }^{*} P=0.0458,{ }^{* *} P=0.0083$. Unless otherwise stated, 1 -way ANOVA statistical analysis was performed on data points; multiple comparisons were performed between indicated columns. All graphs quantify intravital microscopy data. All experiments were repeated 3 times unless otherwise indicated.

(Figure 2, C and D). Similar to PcrV-deficient P. aeruginosa, a subtle increase in $P$. aeruginosa internalization (also not significant) was observed in mice given the anti-PcrV antibody targeting the T3S injectisome (Figure 2, C and D, and Figure 3, A and B).

While no role was noted for T3S in the evasion of neutrophil recognition of $P$. aeruginosa, this may be due to the fact that T3S expression is largely triggered upon contact with host cells or perhaps after phagocytosis and incorporation into a more acidic environment (11). In fact a recent publication observed that
PcrV was important for preventing incorporation of P. aeruginosa into acidic vacuoles in corneal epithelial cells (14). We therefore sought to determine whether blocking PcrV function would result in increased localization of ingested P. aeruginosa into acidified vacuoles in neutrophils. To evaluate this hypothesis, $P$. aeruginos GFP was stained with a $\mathrm{pH}$-sensitive dye (pHrodo Red) that only fluoresces in acidic compartments (pH lower than 6). The very few $P$. aeruginosa that were internalized in neutrophils receiving isotype control antibodies were not in an acidic environment when 
assessed by intravital microscopy (Figure 3, B-D). Moreover, neutrophils from anti-Psl mAb-treated mice caught more bacteria but, again, were not generally observed in a low-pH environment (Figure 3, B-D). In contrast, neutrophils from anti-PcrV-treated mice caught few bacteria; however, most of these internalized bacteria were contained within acidic compartments (Figure 3, B-D). These antibody data were similar to the results seen with the mutant strains of $P$. aeruginosa; more effective neutrophil processing was observed with the $\Delta p c r V P$. aeruginosa than with the WT and $\triangle p s l A$ $P$. aeruginosa within neutrophil acidified phagosomes (Figure $3 \mathrm{E}$ ).

Coordinated targeting of both Psl and PcrV optimizes P. aeruginosa killing. Targeting Psl with therapeutic antibodies led to increased phagocytosis but did not lead to more efficient processing of P. aeruginosa, while targeting PcrV allowed for optimal processing but yielded suboptimal phagocytosis. We therefore tested a recently described bispecific $\mathrm{mAb}$ targeting both Psl and PcrV, which was shown to exhibit synergistic protective activity in an acute pneumonia model (24). Dose-response studies clearly revealed more efficacy of this antibody versus anti-Psl and/or anti-PcrV on an equimolar basis in an acute pneumonia model, but how this antibody functioned remained poorly understood (24). We hypothesized that the dual inhibitory capacity would bind the abundant Psl molecule on extracellular P. aeruginosa, but upon host cell contact and/or internalization of the bacteria, which leads to expression of PcrV, the anti-PcrV motif of the antibody would inhibit the T3S injectisome and allow for proper processing of bacteria. After infection of mice, we observed a striking 8- to 10 -fold increase in neutrophils with captured bacteria in the lungs of animals that received MEDI3902 versus mice that received the IgG control antibody before infection (Figure 4, A and B). These data were confirmed by ex vivo quantification of $P$. aeruginosaGFP-positive neutrophils by flow cytometry from mice treated with anti-PcrV, anti-Psl, and MEDI3902 (Figure 4C). MEDI3902 resulted in the greatest amount of bacterial uptake by neutrophils in the lungs. In addition, we used 3D imaging to confirm that all bacteria were internalized rather than just attached to the surface of neutrophils (Supplemental Video 8). This enhanced recognition of $P$. aeruginosa was specific to the engineered MEDI3902 antibody, as a combination of parenteral anti-PcrV and anti-Psl $\mathrm{mAbs}$, given to mice at molar equivalent dosages, did not cause such a dramatic increase in $P$. aeruginosa uptake by neutrophils (Supplemental Figure 3).

We next examined whether MEDI3902 improved P. aerugino$s a$ localization into acidic vacuoles. Indeed, neutrophils from mice pretreated with MEDI3902 contained significantly more $P$. aeruginosa within acidic compartments than animals receiving control IgG (Figure 4, D and E). There also appeared to be more $P$. aeruginosa in each of the neutrophils in mice that received MEDI3902 even when compared with the anti-Psl mAb. Indeed, neutrophils with multiple internalized bacteria were largely observed only in the MEDI3902-treated mice (Figure 4F). These results suggest that the anti-Psl $\mathrm{mAb}$ alone facilitates neutrophil recognition and phagocytosis, but because of the effective release of T3S effectors once inside the neutrophil, the bacterium avoids acidification, potentially escapes from inside the neutrophil, or prevents further uptake of $P$. aeruginosa by the infected neutrophil. Although difficult to quantify, it was not unusual to see directed migration of neutrophils toward P. aeruginosa in MEDI3902-treated animals, which was not observed in animals treated with the other mAbs (Supplemental Video 7). This may also explain why enhanced sequestration of $P$. aeruginosa by neutrophils was observed in animals treated with bispecific mAb MEDI3902, in comparison with anti-Psl mAb used alone. In mice treated with anti-PcrV alone, so few bacteria were captured by neutrophils that the observation of multiple bacteria per cell was rare (Figure 4F).

The enhanced phagocytosis of bacteria with MEDI3902 or anti-Psl antibody could have been due to direct uptake by the neutrophils or as a consequence of enhanced binding of bacteria to endothelium making more bacteria available for phagocytosis. We therefore assessed the ability of $P$. aeruginosa to bind endothelium in the presence and absence of these antibodies and found no differences in the number of bacteria attached to CD31-positive lung endothelium (endothelial cell-specific marker) (Figure 4G). Consequently, the increased phagocytosis induced by MEDI3902 and the anti-Psl mAb occurred despite no apparent increase in arrest of $P$. aeruginosa on the endothelium.

$C 3$ and FCy receptors are required for optimal anti-Psl- and MEDI3902-mediated neutrophil recognition of $P$. aeruginosa. We identified that C3 was important in mediating neutrophil recognition of Psl-deficient $P$. aeruginosa (Figure 2, D and E). To provide further insight into how MEDI3902 was functioning, the antibody was tested in both $\mathrm{C3}^{-/-}$and Fc $\gamma \mathrm{R}$-deficient $\left(\mathrm{Fcer}^{-\mathrm{g}^{-/}}\right)$mice. Neutrophils of mAb-treated C3-deficient mice no longer showed enhanced phagocytosis of $P$. aeruginos $a$ in any of the therapeutic $\mathrm{mAb}$ treatment groups compared with control IgG (Figure 5A; also compare with Figure 4C). Importantly, the same number of bacteria attached to the endothelium in both C3-deficient and WT mice (Figure 5B). mAb-mediated neutrophil phagocytosis in the lung vasculature of Fc $\gamma \mathrm{R}$-deficient mice was also reduced (Figure $5 \mathrm{C}$ ). These data indicate that both Fc receptors and C3 were essential for uptake of antibody-bound $P$. aeruginosa by lung neutrophils.

MEDI3902 specifically acts on pulmonary neutrophils to increase recognition of $P$. aeruginosa, resulting in decreased bacterial burden and increased survival of mice. We next assessed bacterial organ burden in the lungs of intravenously infected mice that were treated with the therapeutic antibodies. We observed a significant reduction in bacterial burden in lungs 6 hours after infection in anti-PcrV-treated animals compared with the anti-Psl- and control mAb-treated mice and a further 5-fold reduction with MEDI3902 versus the PcrV antibody (Figure 6A). This is consistent with our neutrophil data indicating more efficient phagocytosis and processing of internalized bacteria in MEDI3902-treated animals. Although the neutrophil data were compelling, it is possible that other immune cells also contributed to the improved efficacy of MEDI3902 over Psl antibody treatment. We therefore visualized all immune cells in the lung vasculature using CD45 staining during the first 2 hours of infection and identified 2 other cell types associated with $P$. aeruginosa-GFP that did not stain for Ly6G (Figure 6B). Gating on Ly6 $\mathrm{G}^{-}$cells by flow cytometry, we found a very small population of eosinophils (Ly6G-, Siglec- $\mathrm{F}^{+}$, $\mathrm{CD}_{11 \mathrm{~b}^{+}}$) and monocytes (Ly6G ${ }^{-}$, Siglec-F- $\mathrm{F}^{-} \mathrm{Cd}_{\left.11 \mathrm{~b}^{+}\right)}$that were able to phagocytose $P$. aeruginosa in the presence of either anti-Psl mAb or MEDI3902 (Figure 6C). However, P. aeruginosa uptake by eosinophils and monocytes was minute compared with that by 
A
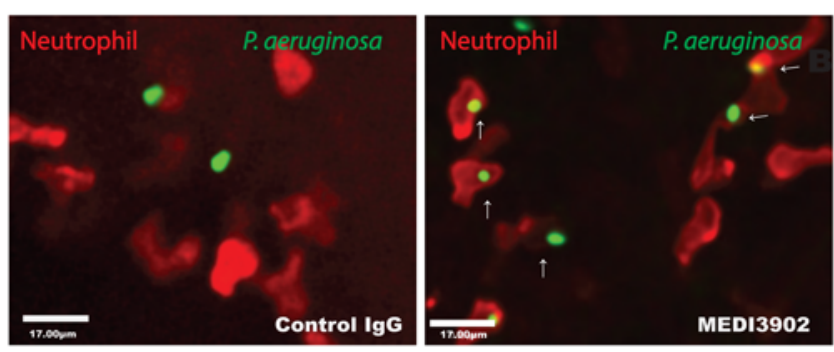

B

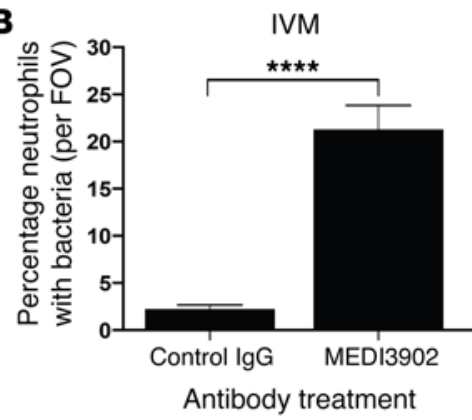

C

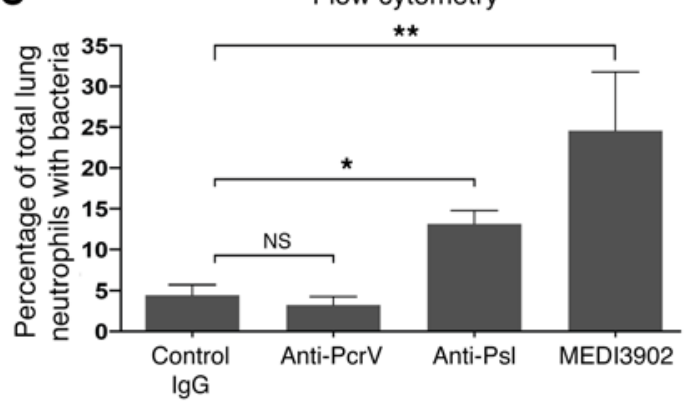

Antibody treatment
D

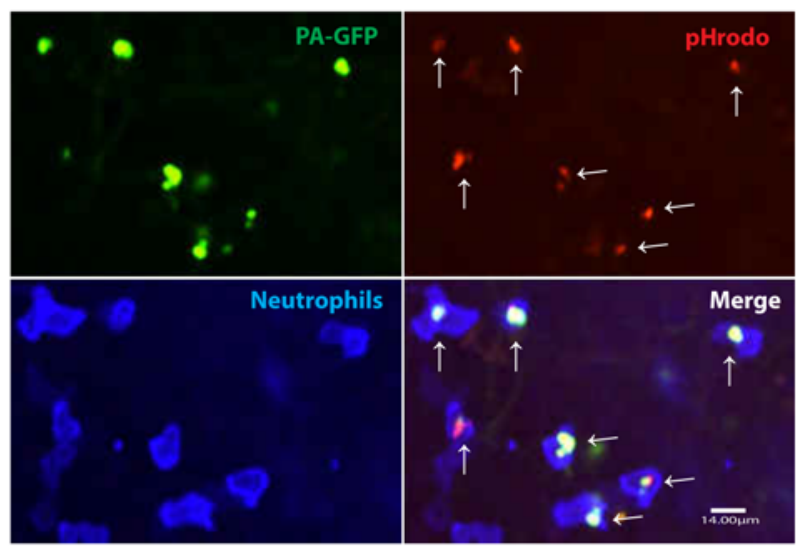

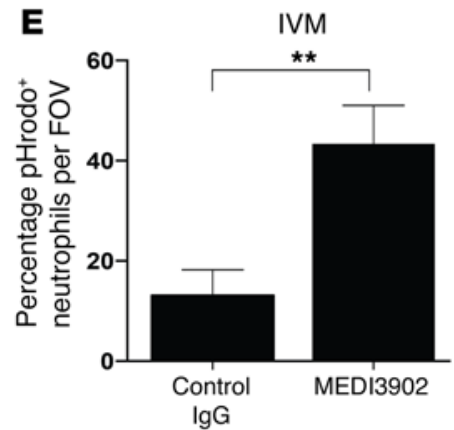

Antibody treatment

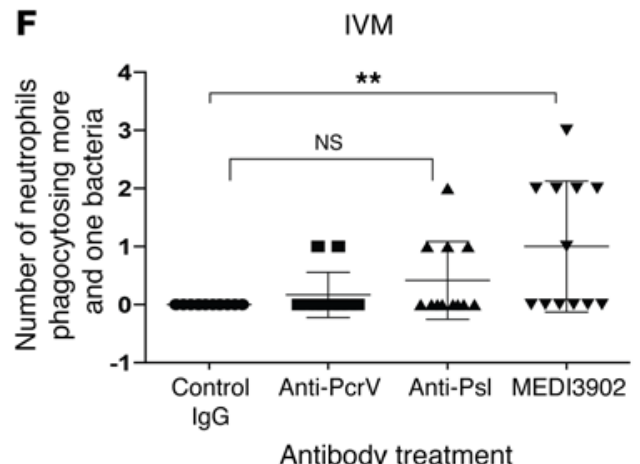

Antibody treatment

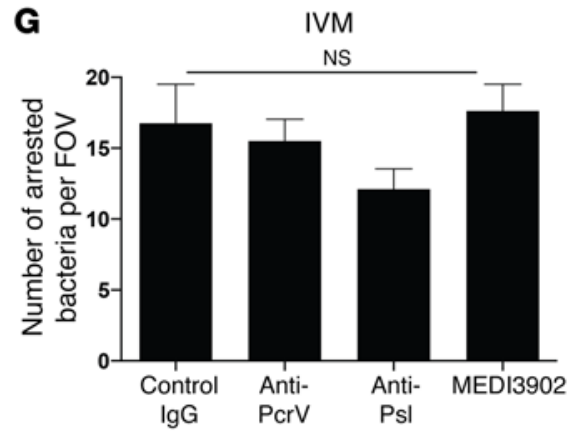

Antibody treatment

Figure 4. Bispecific antibody MEDI3902, targeting PcrV and Psl, promotes enhanced neutrophil capture and lysosomal processing of $P$. aeruginosa. (A) SD-IVM was used to assess the ability of pulmonary intravascular neutrophils (red) to recognize $P$. aeruginosa (green) after i.v. infection in mice prophylactically treated with MEDI3902. White arrows identify neutrophils with bacteria. Representative images were captured 30 minutes after infection. Scale bars: $17 \mu \mathrm{m}$. (B) Quantification of intravital videos: number of neutrophils with bacteria per FOV was assessed in each mouse. $n=4$ per group; 10 -minute videos (3 FOV per mouse). Error bars represent SEM. ${ }^{* * *} P<0.0001$. (C) Ex vivo analysis of neutrophils containing $P$. aeruginosa-GFP by flow cytometry. Lungs were harvested 45 minutes after infection. Neutrophils were gated based on Ly6C (1A8) positive, B220 negative, and CD45 positive. Error bars represent SEM. ${ }^{*} P=0.028,{ }^{* *} P=0.054$. (D) $P$. aeruginosa-GFP stained with pHrodo, a dye that increases in red signal when it is in an acidic environment. Neutrophils, blue; $P$. aeruginosa-GFP stained with pHrodo, green and red. White arrows identify pHrodo-positive cells. Scale bar: $14 \mu \mathrm{m}$. (E) Quantification of intravital videos: number of neutrophils with bacteria in acidified compartments per FOV was assessed in each mouse. $n=4$ per group; 10 -minute videos (3 FOV per mouse). Error bars represent SEM. ${ }^{* *} P=0.004$. (F) Neutrophils in mice prophylactically treated with the respective antibodies were assessed for their ability to engulf more than 1 bacteria. A single neutrophil was tracked for 10 minutes. Error bars represent SEM. ${ }^{* *} P=0.005$. (G) Number of arrested bacteria on endothelium in WT mice. $n=4$ per group. Error bars represent SEM. Unless otherwise stated, 1-way ANOVA statistical analysis was performed on data points; multiple comparisons were performed between indicated columns. Graphs labeled "IVM" quantify intravital microscopy data. All experiments were repeated 3 times unless otherwise indicated.

neutrophils and similar in anti-Psl- and MEDI3902-treated animals, making it unlikely that other immune cells contribute to the efficacy of MEDI3902 (Figure 6, B and C).

Uncontrolled inflammation (cytokine storm) can often contribute to the pathology associated with $P$. aeruginosa infections
(25). Mice treated with antibodies against PcrV and Psl had cytokine and chemokine profiles similar to those of control IgG-treated mice. By contrast, MEDI3902-treated mice exhibited a significant reduction in all of the major proinflammatory cytokines, including TNF- $\alpha$, IL-1 $\beta$, and IL- 6 , and the chemokine KC/GRO compared 
A

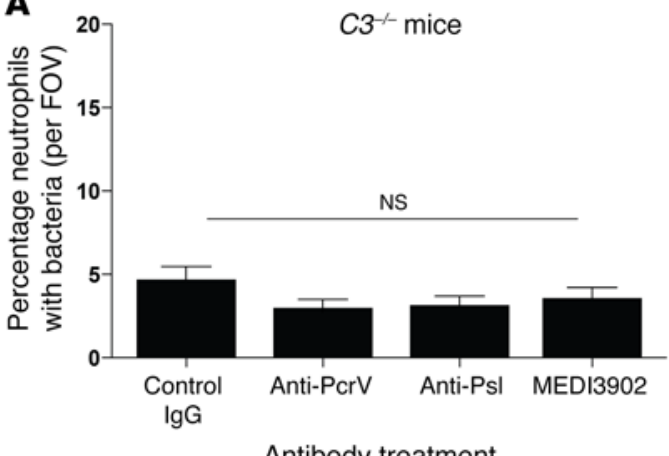

Antibody treatment

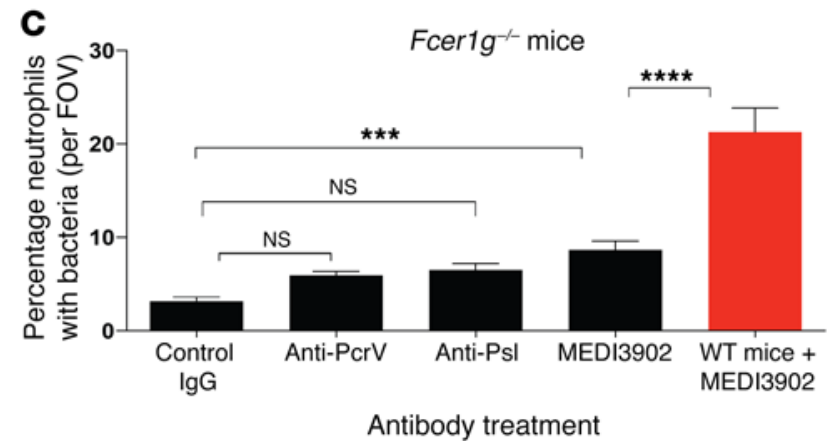

Figure 5. Complement and FcyR play important roles in antibody-mediated recognition of neutrophils. (A) Neutrophils in the lungs of $\mathrm{C} 3-\mathrm{knockout} \mathrm{mice}\left(\mathrm{C3}^{-/-}\right)$prophylactically treated with anti-Pseudomonas mAbs and infected with $P$. aeruginosa were imaged. Quantification of intravital videos: number of neutrophils with bacteria per FOV was assessed in each mouse. $n=4$ per group; 10 -minute videos (3 FOV per mouse). Error bars represent SEM. (B) Number of arrested bacteria on endothelium in WT and $\mathrm{C}^{-/-}$mice. $n=4$ per group; 3 FOV per mouse. Error bars represent SEM. (C) Imaging of neutrophils in the lungs of Fc $\gamma$ receptor-knockout mice (Fcer1 $\left.\mathrm{g}^{-/}\right)$prophylactically treated with anti-Pseudomonas mAbs infected with $P$. aeruginosa. Quantification of intravital videos: percentage of neutrophils with bacteria per FOV was assessed in each knockout mouse in WT mice prophylactically treated with MEDI3902. $n=4$ per group; 3 FOV per mouse; 10 -minute videos. Error bars represent SEM. ${ }^{* * *} P=0.0002,{ }^{* * *} P<0.0001$. Unless otherwise stated, 1-way ANOVA statistical analysis was performed on data points; multiple comparisons were performed between indicated columns. All experiments were repeated 3 times unless otherwise indicated. infections $(14,16)$. Expression of the T3S components and Psl has been shown to be prevalent, with approximately $98 \%$ of isolates capable of expressing 1 or both of these antigens $(25,26)$. Indeed, antibodies targeting 1 or both (24) of these components have shown promising activity against different $P$. aeruginosa strain types in multiple preclinical infection models. However, until now a mechanism of action for how these antibodies were working was poorly understood. Using multilaser spinning-disk microscopy to visualize $P$. aeruginosa infection in the lung and liver, we observed that $P$. aeruginosa evasion and subversion mechanisms predominate in the lung vasculature but not in liver, where intravascular macrophages, known as Kupffer cells, are capable of eradicating many of the bacteria. By initially using isogenic $P$. aeruginosa pslA or pcrV knockout strains stably expressing GFP that were deficient in Psl exopolysaccharide expression or had a dysfunctional T3S injectisome, respectively, we deduced the primary role of these pathogenic factors in vivo. In the lung vasculature, imaging in real time revealed that $P$. aeruginosa uses Psl to impair complement function, thereby remaining invisible to neutrophils and allowing colonization of the organ. By visualizing the host-pathogen response in vivo, we with mice treated with other mAbs (Figure 6D), suggesting uncontrolled infection leading to increased cytokine production is effectively neutralized by MEDI3902 antibody.

We next looked at the efficacy of antibody treatment on disease outcome, in a mouse model of bacteremic infection. In order to do this, we prophylactically treated mice with IgG isotype control or anti-PcrV, anti-Psl, an anti-Psl and anti-PcrV mAb mixture, or MEDI3902 at relative molar concentrations and then infected mice i.v. with $P$. aeruginosa. We found that mice treated with isotype control or anti-Psl antibody succumbed to infection quickly. Mice treated with anti-PcrV had a 50\% increased survival after infection (Figure 6E). This was only slightly increased by administration of both an anti-PcrV and an anti-Psl antibody in tandem (Figure 6E). Importantly, 90\% of mice treated with MEDI3902 survived the infection (Figure 6E).

\section{Discussion}

$P$. aeruginosa is a highly adaptable opportunistic pathogen that can cause life-threatening infections in immunocompromised and critically ill patients. The T3S injectisome and the Psl exopolysaccharide are known to be key factors promoting acute and persistent determined that $P$. aeruginos $a$ effectively evaded neutrophils, suggesting that Psl plays a prominent role in preventing complement fixation in an active infection. We also demonstrate that anti-Psl antibodies acted as opsonins allowing neutrophils in the pulmonary microvasculature to bind and phagocytose $P$. aeruginosa. In addition, our data demonstrate that the mechanism of neutrophil recognition and phagocytosis of $P$. aeruginosa in the lung vasculature required activation of complement. Entirely consistent with these findings is a previous in vitro report stating that higher quantities of complement components were deposited on the Psldeficient strain compared with the isogenic WT parent strain (16).

Interestingly, despite increased uptake of the pathogen in the presence of the anti-Psl mAb, we observed very little phagosomal processing of the bacterium if it possessed a functional T3S. Our data are consistent with previous reports indicating that expression of the T3S system by some $P$. aeruginosa strains helps the bacteria avoid intracellular destruction (14). A study looking at corneal epithelial cells that internalize $P$. aeruginosa showed that the T3S effector ExoS helped the bacteria evade acidic vacuoles once inside the epithelial cell (14). We hypothesize that a similar method of evasion is occurring once neutrophils phagocytose 
A

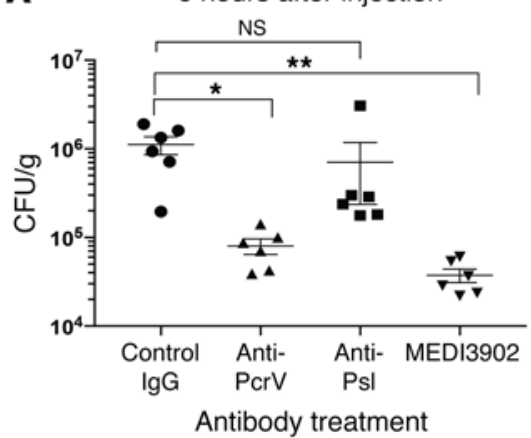

B

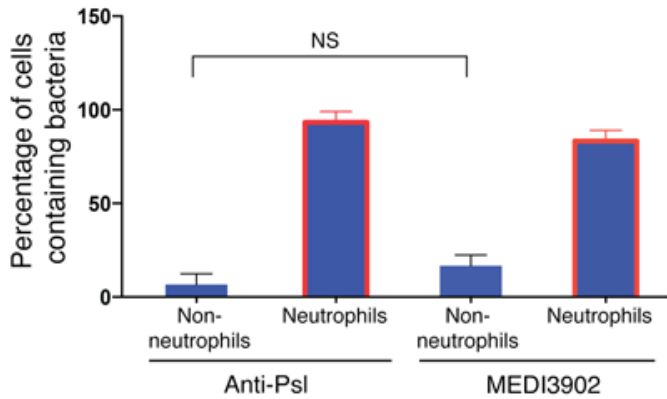

C
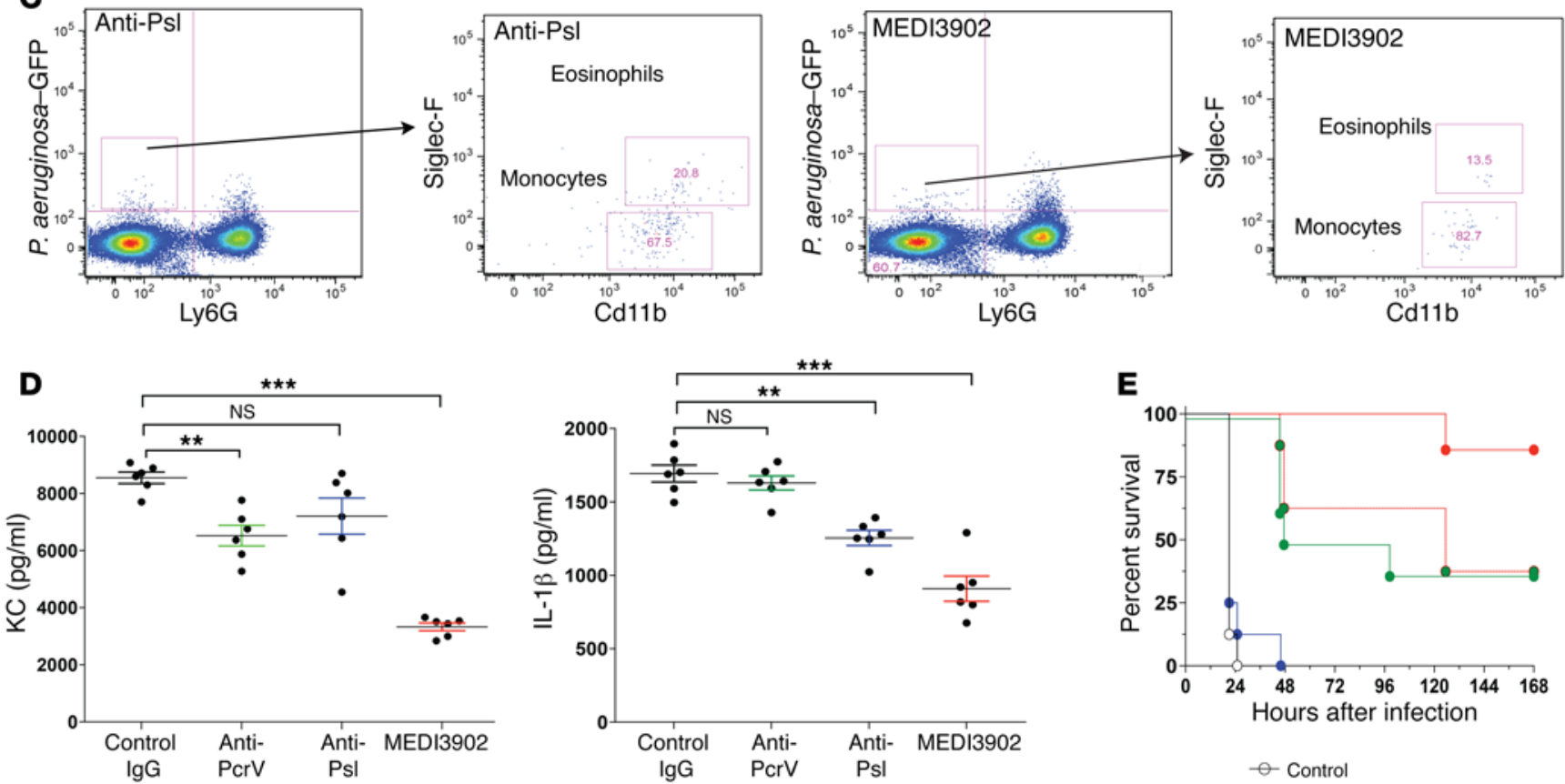

E
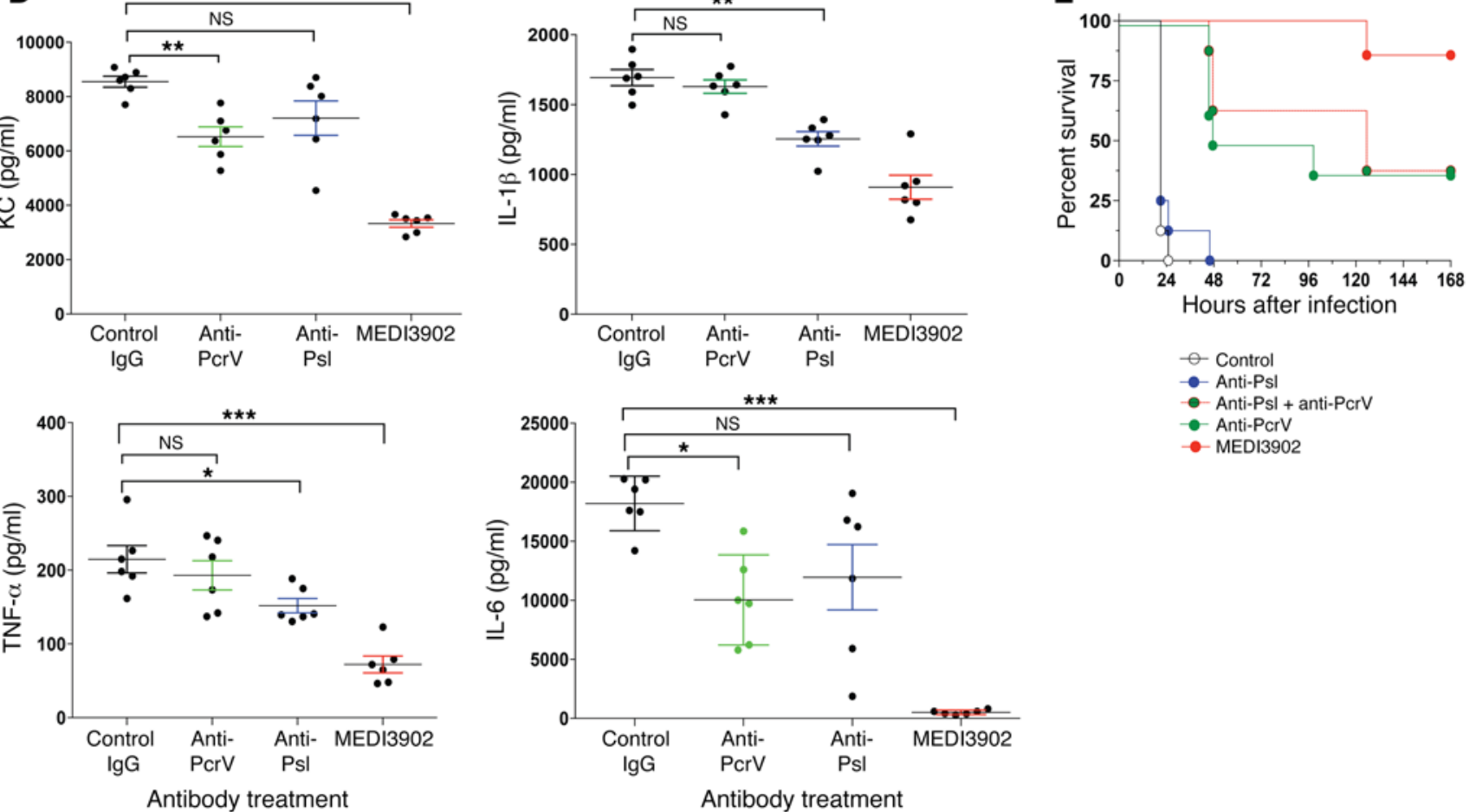

$\theta$ Control

- Anti-Psl

- Anti-Psl + anti-PcrV

- Anti-PcrV

- MEDI3902

Antibody treatment

Figure 6. MEDI3902 increases monocyte/eosinophil recognition of $\boldsymbol{P}$. aeruginosa and decreases lung bacterial load and inflammation. (A) Bacterial CFU were determined at 6 hours after i.v. infection with $P$. aeruginosa in lung after prophylactic antibody treatment. $n=6$. Error bars represent SEM. One-way ANOVA statistical analysis was performed on data points; multiple comparisons were performed between indicated columns. ${ }^{*} P=0.0255,{ }^{* *} P=0.0109$. (B) Mice were stained for CD45 (blue) for all hematopoietic cells and Ly6C (red) for neutrophils. The percentage of neutrophil-negative but CD45-positive cells containing $P$. aeruginosa-GFP was assessed at 1 hour after infection (number of FOV $=3, n=3$ ). Error bars represent SEM. (C) Flow cytometry was performed to identify Ly6G-negative cells that contained $P$. aeruginosa-GFP. Eosinophils are intermediate for Siglec-F and high for CD11b, while monocytes are Siglec-F negative and Cd11b high ( $n=3,3 \mathrm{FOV}$ ). Error bars represent SEM. One-way ANOVA statistical analysis was performed; $P=0.0667$. (D) Whole lung homogenates were used to determine the cytokine/chemokine milieu 6 hours after infection with $P$. aeruginosa. One-way ANOVA analysis was performed on the indicated columns. $P<0.05$ is significant. Error bars represent SEM. ${ }^{*} P=0.0373,{ }^{* *} P=0.0067,{ }^{* *} P=0.0003$. (E) Survival analysis of mice prophylactically treated with therapeutic antibodies and then subsequently infected i.v. with $P$. aeruginosa strain 6077. Mantel-Cox test was performed. There was a significant difference between MEDI3902-treated mice and IgG control mice $(P<0.0001)$. There was a significant difference between antiPcrV-treated mice and IgG control mice $(P<0.0001)$. There was no significant difference between IgG control mice and anti-Psl-treated mice $(P=0.3722)$. Finally, there was a significant difference between mice treated with a mixture of the antibodies (anti-Psl + anti-PcrV) and MED3902-treated mice $(P=0.0491)$. Error bars represent SEM; $n=8$ mice per group. All experiments were repeated 3 times unless otherwise indicated. 


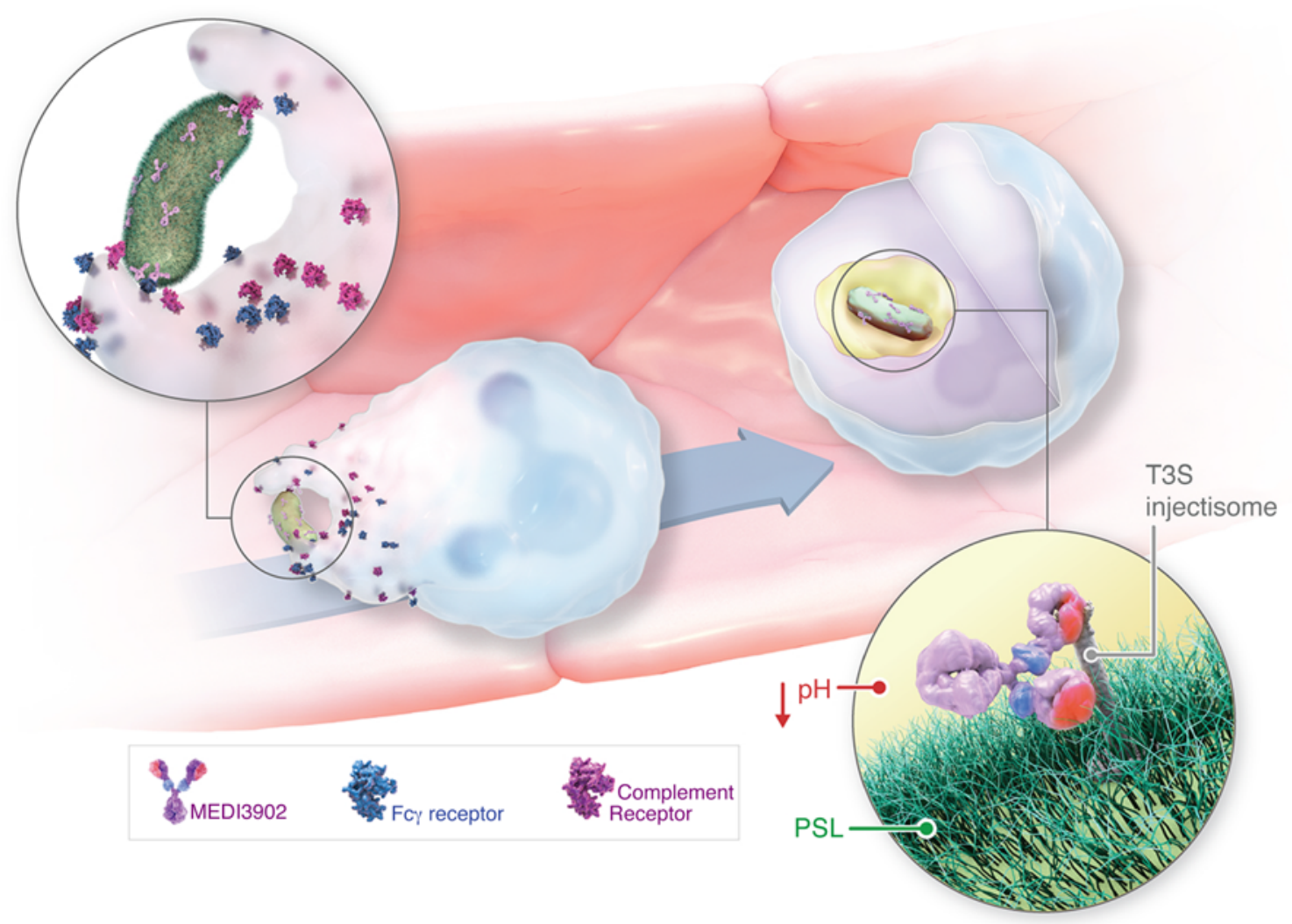

Figure 7. Schematic of pulmonary neutrophil phagocytosis of $\boldsymbol{P}$. aeruginosa in the presence of MEDI3902. WT $P$. aeruginosa avoids detection/phagocytosis by neutrophils in the pulmonary capillaries. P. aeruginosa surface Psl exopolysaccharide inhibits deposition of complement C3b on its surface, cloaking itself from neutrophil recognition. Moreover, phagocytized $P$. aeruginosa secrete effector molecules via the T3S injectisome to hinder acidification of the neutrophil phagolysosome. Deletion of Psl expression ( $\Delta p s / A)$ or targeting of Psl with an anti-PsI mAb increases bacterial recognition and phagocytosis by neutrophils in the lung. However, phagocytized bacteria interfere with acidification of the phagolysosomal compartment. Inhibition of the T3S injectisome, either by infection of mice with T3S injectisome-deficient $P$. aeruginosa $(\Delta p c r V)$ or by treatment with an anti-PcrV mAb alone, does not increase recognition or phagocytosis by neutrophils, but does lead to greater acidification of phagolysosomes in the few neutrophils that engulf bacteria. In contrast, administration of bispecific antibody MEDI3902, which simultaneously targets Psl and PcrV, leads to increased recognition, phagocytosis, and killing of bacteria by neutrophils. This enhanced activity is not observed with a mixture of parental anti-Psl and anti-PcrV mAbs, indicating the necessity of including both specificities on the same mAb molecule. Therefore, the anti-PsI targeting mediated by MEDI3902 facilitates transfer of anti-T3S injectisome activity (via the anti-PcrV arm) within the phagolysosome after phagocytosis, resulting in enhanced compartment acidification and killing of $P$. aeruginosa.

P. aeruginosa. In our model we propose that targeting Psl with the antibody increases recognition of the bacteria by neutrophils (Figure 7). However, inhibition of T3S secretion is required for optimal neutrophil killing of the bacteria (Figure 7). Lung neutrophils from mice receiving anti-PcrV enabled efficient processing of $P$. aeruginosa into low-pH compartments, but the number of neutrophil-bacterial interactions was very low in the absence of antiPsl specificity (Figure 7). Indeed, bispecific mAb MEDI3902, which effectively and simultaneously targets Psl and PcrV, was by far the most effective therapeutic we tested.

In this bacteremic model, we also noted that anti-PcrV and anti-Psl antibodies in combination were not as effective as bispecific mAb MEDI3902, and neutrophils from mice treated with $\mathrm{mAb}$ mixture were not as effective in eradicating $P$. aeruginosa. With the in vivo imaging we were able to visualize which cells in the lung and liver phagocytized P. aeruginosa, and with our ability to visualize capture, phagocytosis, and processing of bacteria inside immune cells, we identify why the antibody combination within a single molecule is superior in its protective activity in compari- son with the individual anti-Psl and anti-PcrV mAbs and the mAb mixture. Having both anti-Psl and anti-PcrV activities on a single molecule allows increased bacterial uptake (mediated by the antiPsl component) by neutrophils while concentrating the colocated anti-PcrV on the bacterium; this was seen by the fact that we could detect antibody in MEDI3902-treated neutrophils but not in antiPcrV-treated neutrophils (Supplemental Figure 4). The anti-PcrV activity of MEDI3902 is thus positioned to inhibit the T3S injectisome as soon as it is expressed, even if it is expressed only after bacteria are ingested intracellularly, thereby resulting in more effective phagosomal processing and eradication of $P$. aeruginosa. These data demonstrate that this is an optimal approach of localizing the antibody for T3S inhibition and may argue that some of the PcrV may be expressed before phagocytosis, with additional PcrV expressed inside the phagolysosomes. Indeed, low $\mathrm{pH}$ has been reported to be a factor that further increases T3S (11).

Patient prognosis is known to be poor upon systemic dissemination of $P$. aeruginosa (26). This bacterium is able to enter the bloodstream of immunocompromised patients, often through i.v. 
catheters during essential invasive interventions. In this study, we use a blood-borne $P$. aeruginosa infection model to assess important immune cell mechanisms in bacterial clearance from the host. In fact, we show that $P$. aeruginosa effectively bound pulmonary endothelium but not hepatic endothelium and evaded lung neutrophils but not liver macrophages (Kupffer cells). Interestingly, this correlated into 2 key phenotypes: (a) greater dissemination of $P$. aeruginosa from the vasculature into the lungs, and (b) increased mortality. In addition to the direct bacterial damage, "friendly fire" resulting from hyperactivation of the immune system in systemic infections causes further injury and organ failure. Multiorgan failure closely precedes death of the host. Interestingly, MEDI3902-treated mice had lower levels of many proinflammatory cytokines and chemokines at 6 hours after infection (Figure 6D) in the lung, which was not observed for the individual anti-PcrV and anti-Psl mAbs in comparison with the control IgG. It could be that MEDI3902 allows for effective clearance of $P$. aeruginosa by pulmonary neutrophils avoiding frustrated phagocytosis and cell lysis, leading to inappropriate release of many of the proteases and other noxious molecules that can injure the host. It could also be the ability of MEDI3902 to inhibit T3S injectisome function more effectively compared with the other antibodies that leads to less cytokine production by the host. It has previously been reported that T3S expression by $P$. aeruginosa is linked to increased IL-1 $\beta$ production (27), which could then induce the production of the many other cytokines and chemokines. In MEDI3902-treated mice, neutrophils appeared more active as evidenced by the ability of neutrophils to engulf multiple bacteria, an outcome not observed in mice treated with anti-Psl, anti-PcrV, or a mAb mixture. Our data suggest that this increased fitness is due to intracellular inhibition of the T3S injectisome.

In conclusion, whether it is during pneumonia leading to bacteremia or bacteremia leading to lung damage, the lung microvasculature is a battleground where neutrophils are critical in containing $P$. aeruginosa lung infections. Here we have visualized the lung of a live mouse during the first few hours of i.v. P. aeruginosa infection. Using intravital microscopy we found that under basal conditions, $P$. aeruginosa expressing Psl evades neutrophil recognition in this organ, and when phagocytosed the pathogen can prevent proper processing into low-pH phagolysosomes via a functional uninhibited T3S system. An antibody directed at both of these molecular targets allows enhanced neutrophil phagocytosis and efficient processing of $P$. aeruginosa and furthermore allows neutrophils to progressively capture and process more than a single bacterium. Precision immunotherapeutic mAbs offer several advantages in comparison with broad-spectrum antibiotics, including extended half-lives without disruption of the beneficial microbiome and without the potential to generate cross-resistance in nontargeted organisms. Given the diversity among $P$. aeruginosa strains, mAb-based approaches against multiple serotypeindependent and highly conserved and pivotal pathogenic mechanisms, such as those targeted herein, may provide significant opportunity for clinical success. Currently, the MEDI3902 mAb is in phase IIb clinical trials where it is given to ventilated patients to prevent the development of pneumonia (17). From the novel mechanisms elucidated in this study, the success of MEDI3902 may also extend beyond treating for bacteremia per se, to treating patients with pneumonia prophylactically to decrease the likelihood of the bacteria entering the systemic circulation and disseminating to other organs.

\section{Methods}

Mice. Six- to 8-week-old C57BL/6 WT male or female mice were purchased from The Jackson Laboratory. All mice were housed under pathogen-free conditions and fed autoclaved rodent feed and water. B6.129S4-C3,tm1Crr./J, referred to as $\mathrm{C}^{-/-}$mice in this paper, were purchased from The Jackson Laboratory (catalog 3641). B6.129P2-Fcer1g ${ }^{\text {tm1Rav }}$ N12, referred to as Fc $\gamma$ R-deficient or Fcer1 $g^{-/}$ mice in this paper, were purchased from Taconic (catalog 583). $C 3^{-1-}$ and $\mathrm{Fcer}_{\mathrm{g}} /{ }^{--}$mice were always used in experiments at 6-8 weeks of age. All experiments were performed in male mice.

Intravital microscopy. Multichannel spinning-disk intravital microscopy was used to image the mouse lungs. We used Ly6G clone $1 \mathrm{~A} 8$ antibodies (Biolegend) to identify neutrophils, F4/80 clone BM8 (eBioscience) to identify Kupffer cells, and CD31 to identify endothelium clone 390 (eBioscience). For intravital experiments we used a very low concentration at $2 \mu \mathrm{g}$ per mouse of each antibody.

Mice were anesthetized $(10 \mathrm{mg} / \mathrm{kg}$ xylazine hydrochloride and $200 \mathrm{mg} / \mathrm{kg}$ ketamine hydrochloride) and body temperature maintained using a heating pad. The right jugular vein was cannulated to administer additional anesthetic and fluorescent dyes.

For lung imaging, the trachea of the mouse was exposed and a small catheter threaded into the trachea, and this was used to attach the mouse to a small rodent ventilator (Harvard Apparatus). The mouse was placed on its side, and 3 ribs were removed in order to expose the lung. The lung was stabilized with a small suction chamber as described previously (28). Suction was maintained at $30 \mathrm{mmHg}$. Images were acquired with an upright microscope (BX51; Olympus) using a $\times 20 / 0.95 \mathrm{~W}$ NA water XLUM Plan F1 objective. The microscope was equipped with a confocal light path (WaveFx; Quorum) based on a modified Yokogawa CSU-10 head (Yokogawa Electric). A 512-by-512-pixel back-thinned electron multiplying charge-coupled device camera (C9100-13; Hamamatsu) was used for fluorescence detection. Simultaneous behavior of multiple cell types in the lung was assessed using 3 laser excitation wavelengths (488, 561, and $635 \mathrm{~nm}$ ) (Cobolt $\mathrm{AB}$ ) in rapid succession and visualized with the appropriate long-pass filters (Semrock). Volocity software (Improvision) was used to acquire and analyze images. For 3D reconstruction, a Z-stack of $1-\mu \mathrm{m}$ slices was captured per field of view for 15 minutes. All intravital data were repeated with 3 strains of $P$. aeruginosa, PAO1, 6206, and 6077 , as well as isogenic mutants deficient in Psl $(\Delta p s l A)$ or T3S $(\Delta p c r V)$ in the 6206 background.

For liver imaging, mice were anesthetized and cannulated as described above, and intravital imaging was performed as previously described $(19,20)$.

Lung image quantification. Ten-minute videos with 3 fields of view were captured for each mouse at a rate of 10 frames per minute. Mice were infected in real time and imaged for 1 hour after infection. Bacterial fluorescence was significantly higher than background autofluorescence of untreated lungs. The total number of neutrophils was quantified for each video, and the number of neutrophils with bacteria was enumerated. The percentage of neutrophils with bacteria was then plotted. Only neutrophils that colocalized with bacteria for more than 30 seconds were considered to have "internalized" the 
bacteria. Average bacterial phagocytosis by neutrophil was calculated for graphs; this was for the first 1-3 hours of infection. All videos were captured at the same speed and exposure time: $492 \mathrm{~ms}$ exposure at 10 frames per minute.

For pHrodo Red experiments, the number of neutrophils with bacteria was calculated, and the percentage of these that had red bacteria was subsequently calculated and graphed.

For Figure 4F, neutrophils with bacteria were tracked for $20 \mathrm{~min}$ utes, and the number of neutrophils containing bacteria was calculated; the percentage of these neutrophils containing bacteria that took up 1 or more other bacteria was then calculated and graphed.

Liver image quantification. For quantification of bacterial phagocytosis in the liver, 5 random fields of view with $\times 10$ objective were selected before injection of bacteria. Acquisition of images was initiated, and after 1 minute of acquiring background images, fluorescent reporter bacteria were injected i.v. via tail vein. The "Find objects" function in Volocity software was used to identify individual captured bacteria by Kupffer cells (F4/80-positive cells in liver), when appropriate autofluorescent spots were subtracted from the final quantification.

For quantification of spinning-disk intravital images 3 hours after infection, computer-generated stitched images of $2 \mathrm{~mm}^{2}$ were generated using the stitched image function in Volocity. Volocity software was used to quantify relative GFP fluorescence as measurement for the presence of $P$. aeruginosa-GFP in the liver. Spinning-disk intravital microscopy images of uninfected mice were used to determine the background fluorescence; $P$. aeruginosa-GFP was quantified with the same settings for all mutants and treatments. All videos and images were captured at the same speed and exposure time: $400 \mathrm{~ms}$ exposure at 3 frames per minute.

Therapeutic antibody treatment. Mice were prophylactically treated with therapeutic MEDI3902 antibody (bispecific mAb targeting Psl and PcrV), individual MEDI3902 parental mAbs targeting either Psl (Psl0096) or PcrV (V2L2-MD), or a parental mAb mixture at a dose of $15 \mathrm{mg} / \mathrm{kg}$ for each mAb through i.p. injection 24 hours before infection.

For fluorescent labeling, antibodies were labeled with Alexa 594 and Alexa 647 fluorophores (Thermo Fisher Scientific). Antibodies were injected i.p. into mice 12 hours before the experiment.

Bacterial growth and infection. P. aeruginosa strains 6077, PAO1GFP, and $6206-\mathrm{GFP}$ were grown overnight at $37^{\circ} \mathrm{C}$ on trypticase soy agar (TSA) plates containing $10 \%$ blood agar. Single colonies were scraped off the plate suspended in PBS to an $\mathrm{OD}_{600}$ of 0.500. Mice were injected i.v. with $1 \times 10^{8} \mathrm{CFU}$ of $P$. aeruginosa and imaged for 1 hour. Videos represent 20 minutes after infection. Quantifications are an average of the number of neutrophils that have phagocytized $P$. aeruginos $a$ over 1 hour. For intravital images, strain 6077 was visualized using Sytox 9 DNA stain. For flow experiments, mouse lungs were harvested 40 minutes after i.v. injection of bacteria. E. coli, S. pneumoniae D39, $S$. aureus MW2, and $P$. aeruginosa 6206 all expressing GFP were administered at a dose of $1 \times 10^{8}$ intravenously. P. aeruginosa-GFP were labeled at $1 \times 10^{8} \mathrm{CFU}$ in $500 \mu \mathrm{l}$ bicarbonate, $100 \mathrm{mM} \mathrm{pH} 8.3$ buffered saline, with $25 \mu \mathrm{g} / \mathrm{ml}$ pHrodo Red and succinimidyl ester for $30 \mathrm{~min}$ utes at room temperature under vigorous agitation. Labeled bacteria were washed twice with PBS and checked for labeling efficiency by flow cytometry or injected i.v. in mice. pHrodo Red, succinimidyl ester, and AF647 NHS ester were obtained from Thermo Fisher Scientific.

Isolation of $P$. aeruginosa mutants and GFP derivatives. P. aeruginosa pslA (Psl-deficient) and pcrV (T3S-deletion) gene knockout strains were constructed in the strain 6206 (cytotoxic, ExoU ${ }^{+}, \mathrm{ExoS}^{-}$) background using an allele replacement strategy as described previously $(29,30)$. Vectors were mobilized from E. coli strain S17.1 into 6206; recombinants were isolated as described previously (31). Gene deletion was confirmed by PCR. GFP derivative strains of $P$. aeruginosa were constructed using the mini-CTX plasmid system containing a ptac-GFP fusion for genomic integration (provided by Dan Wozniak, Ohio State University, Columbus, Ohio, USA). GFP-expression P. aeruginosa were constructed as described previously (32).

Organ burden analyses and quantification of proinflammatory cytokines/chemokines. Seven-week-old C57BL/6 female mice were treated with $\mathrm{mAb}$ (molar equivalent concentrations [150 kDa] of control IgG, or anti-Psl mAb Psl0096, or anti-PcrV mAb V2L2-MD at $11.4 \mathrm{mg} / \mathrm{kg}$ or MEDI3902 [200 kDa] at $15 \mathrm{mg} / \mathrm{kg}$ ) 24 hours before i.v. infection with $P$. aeruginosa strain 6077. Lungs and livers were harvested from animals 6 hours after infection, followed by homogenizing of organs. Homogenized tissues were plated on LB agar plates for identification of bacterial CFU. Supernatants from lung tissue were then analyzed for IL-1 $\beta$, IL-6, KC/GRO, and TNF- $\alpha$ using a multiplex kit (Meso Scale Diagnostics) according to the manufacturer's instructions.

Mouse survival. Seven-week-old C57BL/6 female mice were administered $3.8 \mathrm{mg} / \mathrm{kg}$ of control IgG, anti-Psl, anti-PcrV, mAb mixture $(3.8 \mathrm{mg} / \mathrm{kg}$ of each $\mathrm{mAb})$, or the molar equivalent dosage of MEDI3902 (5 mg/kg) through the i.p. route. Twenty-four hours after prophylactic treatment with therapeutic antibodies, mice were given $1 \times 10^{7} \mathrm{CFU}$ of $P$. aeruginosa strain 6077 intravenously. Mice were monitored hourly for clinical symptoms; mice that lost more than $20 \%$ of their body weight were sacrificed using $\mathrm{CO}_{2}$.

Flow cytometry. Mice were euthanized using $\mathrm{CO}_{2}$. Lungs were perfused with $10 \mathrm{ml}$ of ice-cold saline injected through the right ventricle of the heart. Perfused lungs were removed, minced, and placed in digestion buffer (PBS with $1 \mathrm{mg} / \mathrm{ml}$ collagenase type X1, 60 U DNase). Lungs were digested for 30 minutes and pushed through a $70-\mu \mathrm{M}$ filter. Red blood cells were removed using ACK lysis buffer (Invitrogen), and samples were Fc blocked for 30 minutes. Samples were stained for 30 minutes with indicated antibodies. Samples were run using BD Canto and analyzed using FlowJo software. Flow cytometry was performed with PAO1-GFP and 6206-GFP strains. Bacterial strains used are indicated on the $y$ axes of flow plots. The following antibodies were used in flow analysis: rat anti-mouse CD45 (clone 30-F11; eBioscience), rat IgG2a $\kappa$ isotype control (eBioscience), rat anti-mouse Ly6G (clone 1A8; Biolegend), anti-human/mouse CD45R (B220) (clone RA3-6B2; eBioscience), anti-mouse CD11c (N418; eBioscience), Armenian hamster IgG isotype control, and rat anti-mouse Siglec-F (E50-2440; BD Biosciences). All experiments were repeated with 3 biological samples 3 times. Isotype and fluorescence minus one (FMO) controls were used. Cells were gated based on total CD45-positive population, then on B220-negative and Ly6Gpositive. Within the Ly6G-positive cells, percentage of PAO1-GFPpositive cells was quantified.

Statistics. All error bars represents SEM. One-way ANOVA was performed with multiple comparisons as described in the figure legends. All statistical analyses were performed using the GraphPad Prism program. Unless otherwise indicated, there were 4 mice in each group, and for intravital experiments 3 fields of view were gathered for each mouse. For flow cytometry each group was repeated with 3-4 mice. A $P$ value of less than 0.05 was considered statistically significant. 


\section{Author contributions}

$\mathrm{AT}$ and $\mathrm{AD}$ designed and performed experiments, analyzed data, and wrote the manuscript. BGJS, AEK, GW, PW, MEW, and ASN performed experiments. CKS contributed to experimental design and helped to write the manuscript. BRS contributed to experimental design. AD and PK conceptualized and directed the study, analyzed data, and wrote the manuscript.

\section{Acknowledgments}

The authors thank JoAnn Suzich for critical review of the manuscript. This work was supported by a Canadian Institutes of Health Research (CIHR) Banting Fellowship (to AT) and a CIHR Lung Group Grant for Chronic Inflammation (to PK). BGJS was partially funded by Marie Currie actions FP7-PEOPLE-2013IOF (grant 627575) and Alberta Innovative Health Solutions. We thank Karen Poon from the Snyder Institute Molecular Core for assistance with flow cytometry, and the University of Calgary Live Cell Imaging facility for assistance with multiple microscopy techniques.

Address correspondence to: Paul Kubes, HRIC 4AA16, University of Calgary, 3330 Hospital Drive N.W., Calgary, Alberta, T2N 4N1, Canada. Phone: 403.220.8558; E-mail: pkubes@ucalgary.ca. Or to: Antonio DiGiandomenico, One MedImmune Way, Gaithersburg, Maryland 20878, USA. Phone: 301.398.2401; E-mail: DiGiandomenicoA@MedImmune.com.
1. Sandiumenge A, Rello J. Ventilator-associated pneumonia caused by ESKAPE organisms: cause, clinical features, and management. Curr Opin Pulm Med. 2012;18(3):187-193.

2. Kang CI, et al. Pseudomonas aeruginosa bacteremia: risk factors for mortality and influence of delayed receipt of effective antimicrobial therapy on clinical outcome. Clin Infect Dis. 2003;37(6):745-751.

3. Guillamet CV, Vazquez R, Noe J, Micek ST, Kollef $\mathrm{MH}$. A cohort study of bacteremic pneumonia: The importance of antibiotic resistance and appropriate initial therapy? Medicine (Baltimore). 2016;95(35):e4708.

4. Tam VH, et al. Prevalence, resistance mechanisms, and susceptibility of multidrugresistant bloodstream isolates of Pseudomonas aeruginosa. Antimicrob Agents Chemother. 2010;54(3):1160-1164.

5. Kunz AN, Brook I. Emerging resistant Gramnegative aerobic bacilli in hospital-acquired infections. Chemotherapy. 2010;56(6):492-500.

6. Micek ST, et al. An international multicenter retrospective study of Pseudomonas aeruginosa nosocomial pneumonia: impact of multidrug resistance. Crit Care. 2015;19:219.

7. Lovewell RR, Patankar YR, Berwin B. Mechanisms of phagocytosis and host clearance of Pseudomonas aeruginosa. Am J Physiol Lung Cell Mol Physiol. 2014;306(7):L591-L603.

8. Turner KH, Everett J, Trivedi U, Rumbaugh KP, Whiteley M. Requirements for Pseudomonas aeruginosa acute burn and chronic surgical wound infection. PLoS Genet 2014;10(7):e1004518.

9. Gellatly SL, Hancock RE. Pseudomonas aeruginosa: new insights into pathogenesis and host defenses. Pathog Dis. 2013;67(3):159-173.

10. Hauser AR. The type III secretion system of Pseudomonas aeruginosa: infection by injection. Nat Rev Microbiol. 2009;7(9):654-665.

11. Galle M, Carpentier I, Beyaert R. Structure and function of the Type III secretion system of Pseudomonas aeruginosa. Curr Protein Pept Sci. 2012;13(8):831-842.
12. Vance RE, Rietsch A, Mekalanos JJ. Role of the type III secreted exoenzymes S, T, and Y in systemic spread of Pseudomonas aeruginosa PAO1 in vivo. Infect Immun. 2005;73(3):1706-1713.

13. Duncan MC, Linington RG, Auerbuch V. Chemical inhibitors of the type three secretion system: disarming bacterial pathogens. Antimicrob Agents Chemother. 2012;56(11):5433-5441.

14. Heimer SR, Evans DJ, Stern ME, Barbieri JT, Yahr T, Fleiszig SM. Pseudomonas aeruginosa utilizes the type III secreted toxin ExoS to avoid acidified compartments within epithelial cells. PLOS ONE. 2013;8(9):e73111.

15. Limoli DH, Jones CJ, Wozniak DJ. Bacterial extracellular polysaccharides in biofilm formation and function. Microbiol Spectr. 2015;3(3):MB-0011-2014.

16. Mishra M, et al. Pseudomonas aeruginosa Psl polysaccharide reduces neutrophil phagocytosis and the oxidative response by limiting complement-mediated opsonization. Cell Microbiol. 2012;14(1):95-106.

17. MedImmune LLC. Effort to prevent nosocomial pneumonia caused by Pseudomonas aeruginosa in mechanically ventilated subjects (EVADE). NIH Web site. https://clinicaltrials.gov/ct2/show/ NCT02696902?term=MEDI3902\&rank=2. Published February 11, 2016. Accessed March 15, 2017

18. Protzer U, Maini MK, Knolle PA. Living in the liver: hepatic infections. Nat Rev Immunol. 2012;12(3):201-213

19. Zeng Z, Surewaard BG, Wong CH, Geoghegan JA, Jenne CN, Kubes P. CRIg Functions as a macrophage pattern recognition receptor to directly bind and capture blood-borne gram-positive bacteria. Cell Host Microbe. 2016;20(1):99-106.

20. Surewaard BG, et al. Identification and treatment of the Staphylococcus aureus reservoir in vivo. JExp Med. 2016;213(7):1141-1151.

21. Sawa T, et al. Active and passive immunization with the Pseudomonas $\mathrm{V}$ antigen protects against type III intoxication and lung injury. Nat Med 1999;5(4):392-398.

22. Schneberger D, Aharonson-Raz K, Singh B. Pulmonary intravascular macrophages and lung health: what are we missing? Am J Physiol Lung Cell Mol Physiol. 2012;302(6):L498-L503.

23. Kreisel D, et al. In vivo two-photon imaging reveals monocyte-dependent neutrophil extravasation during pulmonary inflammation. Proc Natl Acad Sci U S A. 2010;107(42):18073-18078.

24. DiGiandomenico A, et al. A multifunctional bispecific antibody protects against Pseudomonas aeruginosa. Sci Transl Med. 2014;6(262):262ra155

25. D'Elia RV, Harrison K, Oyston PC, Lukaszewski RA, Clark GC. Targeting the "cytokine storm" for therapeutic benefit. Clin Vaccine Immunol. 2013;20(3):319-327.

26. Faure K, et al. Effects of monoclonal anti-PcrV antibody on Pseudomonas aeruginosa-induced acute lung injury in a rat model. J Immune Based Ther Vaccines. 2003;1(1):2

27. Marion CR, et al. Chitinase 3-like 1 (Chil1) regulates survival and macrophage-mediated interleukin-1 $\beta$ and tumor necrosis factor alpha during pseudomonas aeruginosa pneumonia. Infect Immun. 2016;84(7):2094-2104.

28. Looney MR, Thornton EE, Sen D, Lamm WJ, Glenny RW, Krummel MF. Stabilized imaging of immune surveillance in the mouse lung. Nat Methods. 2011;8(1):91-96.

29. Schweizer HP. Allelic exchange in Pseudomonas aeruginosa using novel ColE1-type vectors and a family of cassettes containing a portable oriT and the counter-selectable Bacillus subtilis sacB marker. Mol Microbiol. 1992;6(9):1195-1204.

30. Schweizer HD. Small broad-host-range gentamycin resistance gene cassettes for site-specific insertion and deletion mutagenesis. BioTechniques. 1993;15(5):831-834.

31. Hoang TT, Karkhoff-Schweizer RR, Kutchma AJ, Schweizer HP. A broad-host-range Flp-FRT recombination system for site-specific excision of chromosomally-located DNA sequences: appli cation for isolation of unmarked Pseudomonas aeruginosa mutants. Gene. 1998;212(1):77-86.

32. Wyckoff TJ, Wozniak DJ. Transcriptional analysis of genes involved in Pseudomonas aeruginosa biofilms. Meth Enzymol. 2001;336:144-151. 